\title{
Role of the temporal pole in temporal lobe epilepsy seizure networks: an intracranial electrode investigation
}

\author{
Taylor J. Abel, MD, ${ }^{1}$ Royce W. Woodroffe, MD, ${ }^{1}$ Kirill V. Nourski, MD, PhD, ${ }^{1}$ Toshio Moritani, MD, ${ }^{2}$ \\ Aristides A. Capizzano, MD, ${ }^{2}$ Patricia Kirby, MD, ${ }^{3}$ Hiroto Kawasaki, MD, ${ }^{1}$ Matthew Howard III, MD, ${ }^{1}$ \\ and Mary Ann Werz, MD ${ }^{4}$
}

Departments of ${ }^{1}$ Neurosurgery, ${ }^{2}$ Radiology, ${ }^{3}$ Pathology, and ${ }^{4}$ Neurology, University of lowa Hospitals and Clinics, lowa City, lowa

\begin{abstract}
OBJECTIVE A convergence of clinical research suggests that the temporal pole (TP) plays an important and potentially underappreciated role in the genesis and propagation of seizures in temporal lobe epilepsy (TLE). Understanding its role is becoming increasingly important because selective resections for medically intractable TLE spare temporopolar cortex (TPC). The purpose of this study was to characterize the role of the TPC in TLE after using dense electrocorticography $(E C O G)$ recordings in patients undergoing invasive monitoring for medically intractable TLE.
\end{abstract}

METHODS Chronic ECoG recordings were obtained in 10 consecutive patients by using an array customized to provide dense coverage of the TP as part of invasive monitoring to localize the epileptogenic zone. All patients would eventually undergo cortico-amygdalohippocampectomy. A retrospective review of the patient clinical records including ECoG recordings, neuroimaging studies, neuropathology reports, and clinical outcomes was performed.

RESULTS In 7 patients (70\%), the TP was involved at seizure onset; in 7 patients (70\%), there were interictal discharges from the TP; and in 1 case, there was early spread to the TP. Seizure onset in the TP did not necessarily correlate with preoperative neuroimaging abnormalities of the TP.

CONCLUSIONS These data demonstrate that TPC commonly plays a crucial role in temporal lobe seizure networks. Seizure onset from the TP would not have been predicted based on available neuroimaging data or interictal discharges. These findings illustrate the importance of thoroughly considering the role of the TP prior to resective surgery for TLE, particularly when selective mesial resection is being considered.

https://thejns.org/doi/abs/10.3171/2017.3.JNS162821

KEY WORDS seizures; anterior temporal lobe; epilepsy surgery; temporal lobe epilepsy; electrocorticography

$\mathrm{T}$ EMPORAL lobe epilepsy (TLE) is the most common type of surgically remediable intractable epilepsy in adults. Of the appropriately selected patients with medically intractable TLE who undergo cortico-amygdalohippocampectomy (CAH), 60\%-80\% demonstrate longterm seizure freedom and up to $95 \%$ have improvement in seizure control. ${ }^{16,23,34}$ In a significant proportion of patients with TLE, seizures arise from the mesial temporal lobe, which is associated with a syndrome defined as mesial temporal lobe epilepsy (MTLE). This syndrome is typified by neuroimaging findings of mesial temporal sclerosis (MTS), a characteristic clinical semiology, neuropsycho- logical evidence of mesial temporal dysfunction, and anterior and mid-inferomedial temporal ictal and interictal discharges on scalp electroencephalography (EEG), ${ }^{9,25} \mathrm{~Pa}-$ tients with findings characteristic of MTLE may be candidates for more selective mesial temporal resections such as selective amygdalohippocampectomy $(\mathrm{SAH})$ or the more recent laser interstitial thermal therapy (LITT). ${ }^{11,15,19,35}$ Notably, the indications to undergo CAH or a more selective mesial approach such as SAH or LITT vary widely among epilepsy surgery centers. Furthermore, while there is clear evidence that mesial resection is effective for achieving seizure freedom in appropriately selected patients, 3,32

ABBREVIATIONS ATL = anterior temporal lobe; $\mathrm{CAH}=$ cortico-amygdalohippocampectomy; $\mathrm{DNT}=$ dysembryoplastic neuroepithelial tumor; $\mathrm{ECoG}=$ electrocorticography; EEG = electroencephalography; IED = interictal epileptiform discharge; ILAE = International League Against Epilepsy; LITT = laser interstitial thermal therapy; MTLE = mesial temporal lobe epilepsy; MTS = mesial temporal sclerosis; SAH = selective amygdalohippocampectomy; SEEG = stereo-EEG; TLE = temporal lobe epilepsy; TP = temporal pole; TPC = temporopolar cortex.

SUBMITTED November 10, 2016. ACCEPTED March 23, 2017.

INCLUDE WHEN CITING Published online October 13, 2017; DOI: 10.3171/2017.3.JNS162821. 
TABLE 1. Clinical characteristics, surgery, and seizure outcome

\begin{tabular}{|c|c|c|c|c|c|c|c|c|c|c|c|}
\hline $\begin{array}{l}\text { Case } \\
\text { No./ } \\
\text { Group }\end{array}$ & Sex & $\begin{array}{l}\text { Age } \\
\text { (yrs) }\end{array}$ & $\begin{array}{l}\text { Febrile } \\
\text { Szs }\end{array}$ & $\begin{array}{l}\text { Age at } \\
\text { Epilepsy } \\
\text { Onset } \\
\text { (yrs) }\end{array}$ & $\begin{array}{l}\text { Epilepsy } \\
\text { Laterality }\end{array}$ & $\begin{array}{c}\text { Surgical } \\
\text { Intervention }\end{array}$ & Aura & $\begin{array}{l}\text { Scalp } \\
\text { EEG } \\
\text { IEDs* }^{*}\end{array}$ & $\begin{array}{c}\text { Scalp EEG } \\
\text { Seizure Pattern† }\end{array}$ & $\begin{array}{l}\text { Postop } \\
\text { Engel } \\
\text { Outcome } \\
\text { Class }\end{array}$ & $\begin{array}{c}\mathrm{FU} \\
\text { (mos) }\end{array}$ \\
\hline $1-A$ & M & 21 & No & 16 & Rt & $\mathrm{Rt} \mathrm{CAH}$ & Deja vu & $\mathrm{F} 8=\mathrm{T} 4$ & $\begin{array}{l}\text { Several F8 maximal sharp } \\
\text { waves, then rt fronto-cen- } \\
\text { tro-temporal theta/delta }\end{array}$ & I & 2 \\
\hline $2-A$ & $\mathrm{M}$ & 29 & Yes & 18 & $\mathrm{Lt}$ & Lt CAH & Rt foot tingling & T3 or SP1 & SP1 sharp waves \& delta & 1 & 40 \\
\hline $3-A$ & $\mathrm{~F}$ & 50 & No & 1 & $\mathrm{Lt}$ & $\begin{array}{l}\text { Lt CAH + le- } \\
\text { sionectomy }\end{array}$ & None & $\mathrm{F} 8=\mathrm{T} 4$ & Artifact obscured & ॥ & 21 \\
\hline $4-\mathrm{A}$ & $\mathrm{M}$ & 31 & No & 23 & Rt & Rt CAH & $\begin{array}{l}\text { Nausea, hear- } \\
\text { ing things }\end{array}$ & F8 or T4 & $\begin{array}{l}\text { Rt lateralized or regional } \\
\text { temporal delta }\end{array}$ & II & 43 \\
\hline $5-\mathrm{A}$ & M & 26 & Yes & 21 & Rt & $\begin{array}{l}\mathrm{Rt} \mathrm{CAH}+\mathrm{OF} \\
\text { resection }\end{array}$ & Dizzy & $\mathrm{FP} 2>\mathrm{F} 8$ & Artifact obscured & III & 8 \\
\hline $6-A$ & M & 31 & No & 24 & $\mathrm{Lt}$ & Lt CAH & $\begin{array}{l}\text { Smell, warmth, } \\
\text { tingling }\end{array}$ & T3 & Lt lateralized polyfrequency & I & 46 \\
\hline $7-A$ & $\mathrm{M}$ & 47 & No & 35 & $\mathrm{Lt}$ & Lt CAH & Abdominal & $\mathrm{F} 7>\mathrm{T} 3$ & Lt regional temporal theta & I & 27 \\
\hline $8-B$ & M & 26 & No & 21 & Rt & Rt CAH & $\begin{array}{l}\text { Present but not } \\
\text { described }\end{array}$ & T6 & $\begin{array}{l}\text { T6 \& right fronto-temporal } \\
\text { alpha }\end{array}$ & II & 57 \\
\hline $9-B$ & $\mathrm{M}$ & 35 & No & 33 & $\mathrm{Lt}$ & Lt CAH & Abdominal & T3 > T5 (\& SP1) & Lt regional temporal theta & I & 32 \\
\hline $10-B$ & $M$ & 51 & No & 33 & $\mathrm{Lt}$ & Lt CAH & None & T3 (\& SP1) & Lt regional temporal theta & I & 38 \\
\hline
\end{tabular}

some studies suggest lower seizure freedom after selective mesial resection, particularly after LITT. ${ }^{23,35}$ Patients with incomplete improvement after selective mesial resection or ablation probably have extrahippocampal seizure genesis sites. Understanding which patients have extrahippocampal seizure genesis is key to determining the correct resection strategy for patients with TLE.

The temporal pole (TP) has been proposed as an important site of seizure genesis within temporal lobe seizure networks. ${ }^{9,21}$ Intracerebral studies with concurrent recording of the hippocampus and TP suggest that the TP becomes involved in seizures either concurrent with or before the hippocampus in approximately $50 \%$ of patients. ${ }^{9}$ However, exactly what role the temporopolar cortex (TPC) plays in temporal lobe seizure networks and TLE remains poorly understood. One barrier to understanding the role of the TP in seizure networks is the tendency of many investigators to place relatively few electrodes on the TP during intracranial recording studies used to localize seizures. ${ }^{2}$ Standard electrocorticography (ECoG) implantation strategies typically include an anteromedial strip electrode $^{12}$ or temporopolar strip electrodes, which both leave much of the TP uncovered. Alternatively, groups using stereo-EEG (SEEG) may place only one depth electrode in the TP. ${ }^{9}$ With any of these intracranial recording strategies, coverage of the TP is relatively sparse, and it is possible that ictal onset in the TP could be missed.

Given the relative lack of information on the role of the TP in TLE, the purpose of this study was to examine the role of the TPC in temporal lobe seizure networks. We hypothesized, based on previous studies, ${ }^{9}$ that the TP would be involved in seizure onset regardless of whether the neuroimaging data had supported a role for the TP. To test our hypothesis, we reviewed recordings that had been obtained with an electrode array specialized to provide high-density recordings of the TP in 10 consecutive patients who ultimately underwent $\mathrm{CAH} .{ }^{2}$

\section{Methods}

\section{Patients and Preoperative Evaluation}

Ten consecutive patients who had undergone $\mathrm{CAH}$ after dense intracranial recording of the anterior temporal lobe (ATL) were included in this study (Table 1). Each subject had been shown to have medically intractable epilepsy following multiple medication trials and thus was a candidate for surgical evaluation. To maintain the most homogeneous study population possible, each patient had the following characteristics: 1) seizures of temporal lobe origin, although the epileptogenic zone could not be satisfactorily estimated using noninvasive methods, 2) dense ATL recording with either a specialized ATL recording array (see below) or 2 dedicated ATL strip electrodes in addition to a standard anteromedial strip electrode, ${ }^{12}$ and 3) ultimately underwent CAH. Thus, patients with spatially sparse ATL recording limited to a standard anteromedial strip electrode or patients who had undergone lesionectomy without $\mathrm{CAH}$ were not included.

The study group consisted of 9 men and 1 woman, with a mean age of 34.7 years (range 21-51 years). Epilepsy was lateralized to the left hemisphere in 6 patients and the right hemisphere in the other 4. Age at onset ranged from 1 to 35 years. Two patients (Cases 2 and 5) had a history of febrile seizures. 

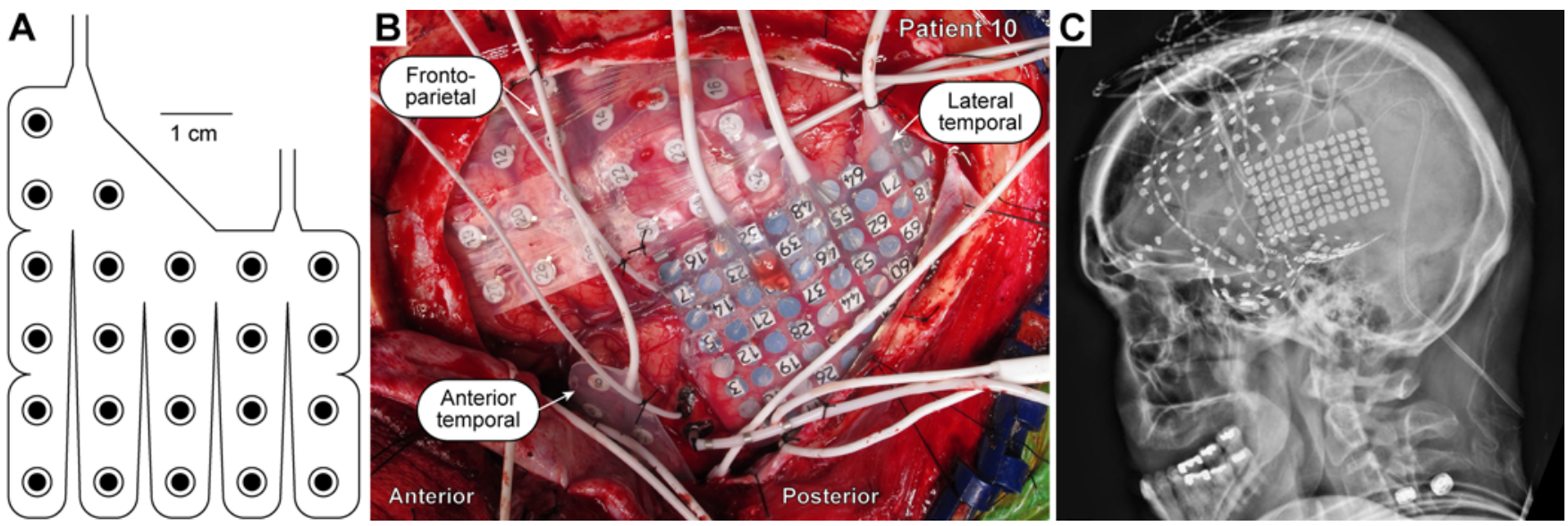

FIG. 1. Intracranial recording from the ATL. A: Schematic of a customized 23-contact ATL electrode array. B: Intraoperative photograph depicting placement of the recording arrays, including the ATL array. C: Radiograph obtained in a patient implanted with the ATL array.

After the determination of medically intractable disease, each subject underwent preoperative epilepsy surgery workup per the University of Iowa Comprehensive Epilepsy Center protocol. This included 3-T MRI with a high-resolution epilepsy protocol, interictal FDG-PET, inpatient video EEG monitoring, neuropsychological examination, and, in some cases, Wada testing. The epileptogenic zone could not be satisfactorily estimated using noninvasive methods in any of the subjects; therefore, further evaluation was performed using intracranial grid, strip, and depth electrodes in each subject (see below).

Ultimately, after localization of the ictal onset zone and estimation of the epileptogenic zone, each patient underwent a tailored $\mathrm{CAH}$. In addition to the $\mathrm{CAH}$, one patient had a frontal lesionectomy (Case 3) and another patient had an orbitofrontal resection (Case 5), which were both deemed necessary on the basis of intracranial recordings. All CAHs were performed by a single surgical team (H.K. and M.H.). The CAH technique involved an approach through the middle temporal gyrus with resection of the hippocampus, amygdala, and parahippocampal gyrus. A tailored resection of the hippocampus was guided by intraoperative ECoG in each patient. After resection of the mesial structures, the ATL and TPC were disconnected in each case. The posterior margin of the anterior temporal disconnection was determined by extraoperative ECoG in each case.

\section{Intracranial Electrode Implantation and Recording}

Chronic intracranial recordings were performed in all patients to estimate the extent of the epileptogenic zone in the temporal lobe and to determine whether extratemporal brain structures were involved. Thus, intracranial recordings in all cases included grid, strip, and depth electrodes. To place electrodes, a frontotemporal craniotomy was performed on the side determined by noninvasive diagnostic tests to contain the region of seizure onset. In each case electrode coverage was determined on clinical grounds based on findings from seizure semiology, MRI, video EEG, and FDG-PET. Frameless stereotaxy was used to place depth electrodes in all subjects, and each subject had at least a hippocampal and an amygdala depth electrode placed.

All patients in the study had dense subdural electrode coverage of the ATL, including the TP. Recently, we developed a specialized electrode array that provides dense and consistent subdural electrode coverage from the TP for clinical recording purposes. ${ }^{2}$ The design and implantation of this array has been described elsewhere ${ }^{2}$ and is summarized in Fig. 1. Thus, our criterion for dense recordings of the TP was implantation of a customized TP electrode array or placement of at least 3 strip electrodes covering the TP (1 standard anteromedial strip electrode and 2 ATL strip electrodes).

Anatomical definitions of the TP vary widely throughout the literature. ${ }^{1,8,9,14}$ For our purposes in this study, we considered the TP to be that region of the ATL that is anterior to the rhinal sulcus. ${ }^{9}$ We chose this definition because it is consistent with previous clinical studies of the electrophysiological properties of the TP in TLE patients.

Chronic recording was performed over the course of 1-4 weeks. The duration of monitoring was determined based on the time necessary to record ictal events. Depending on which imaging data were available for each patient, electrodes were localized to the brain surface using a combination of intraoperative photographs, axial CT images with 1-mm slices, posteroanterior and lateral skull radiographs, and MRI studies.

\section{Clinical and Electrophysiological Seizure Analysis}

The clinical and electrophysiological characteristics of each seizure were monitored with concurrent video and intracranial recordings. After electrode implantation, recording commenced immediately and continued without interruption until the resection period. All intracranial recording and seizure semiology data were analyzed by a board-certified clinical electrophysiologist and epileptologist (M.A.W.).

Each patient's seizure network was characterized based on involvement of the TP. According to an analysis of multiple seizures, each patient's seizure network was classified as one of the following: ictal onset involving the TP (Group 
TABLE 2. Interictal epileptiform discharges, seizure onsets, and seizure spread patterns in 10 patients

\begin{tabular}{|c|c|c|c|c|c|c|c|c|c|}
\hline \multirow{2}{*}{$\begin{array}{c}\text { Case No./ } \\
\text { Group }\end{array}$} & \multirow{2}{*}{$\begin{array}{c}\text { TP } \\
\text { IEDs }\end{array}$} & \multicolumn{4}{|c|}{ Intracranial EEG Sz Onset } & \multicolumn{3}{|c|}{ Intracranial EEG Sz Propagation* } & \multirow{2}{*}{$\begin{array}{c}\text { Subclinical } \\
\text { TP }\end{array}$} \\
\hline & & TP & HPC & Amygdala & Other Temporal Neocortex & TP & HPC & Amygdala & \\
\hline $1-\mathrm{A}$ & + & + & - & - & - & NA & - & 10 secs & NA \\
\hline $2-A$ & + & + & - & - & + & NA & - & - & NA \\
\hline $3-A$ & - & + & + & + & - & NA & NA & NA & NA \\
\hline $4-\mathrm{A}$ & + & + & - & - & - & NA & - & - & NA \\
\hline $5-A$ & - & + & + & - & - & NA & NA & - & NA \\
\hline $6-A$ & + & + & - & - & + & NA & - & - & NA \\
\hline $7-A$ & + & + & - & - & + & NA & 10 secs & 10 secs & - \\
\hline $8-B$ & - & - & - & - & + & 5 secs & - & 4-10 secs & + \\
\hline $9-B$ & + & - & - & + & - & - & - & NA & - \\
\hline $10-B$ & + & - & - & + & - & - & - & NA & - \\
\hline
\end{tabular}

$+=$ present or involved; $-=$ not present or not involved; HPC = hippocampus; NA = not applicable.

* Listed times indicate latency to involvement in seizure.

A) or ictal onset outside the TP (Group B) in the hippocampus, amygdala, or other TPC. Patients with concurrent ictal onset in the TP and other structures (for example, the hippocampus) were also classified as having ictal onset in the TP. The time from seizure onset to the onset of TP involvement was documented for characteristic seizures. To characterize the relationship between seizure onset in the TP and other temporal lobe structures (for example, hippocampus, amygdala, or temporal neocortex), onsets from these structures were also documented.

\section{Neuroimaging and Neuropathological Analysis}

Clinical and electrophysiological data were correlated to available neuroimaging and neuropathological specimens. Preoperative MRI was retrospectively analyzed by 2 board- and subspecialty (certificate of added qualification [CAQ])-certified neuroradiologists (T.M. and A.A.C.) who were blinded to all other clinical data (that is, semiology, EEG, and PET). During MRI analysis, the neuroradiologists investigated the presence of 1) ATL abnormalities, 2) hippocampal sclerosis, and 3) anatomical lesions anywhere in the brain. Interpretation of neuropathological specimens was performed by 1 neuropathologist (P.K.) to determine the etiology of epilepsy. When appropriate, the International League Against Epilepsy (ILAE) classification of cortical dysplasia was applied in the pathological diagnosis. ${ }^{5}$

\section{Results}

\section{Electrophysiological Results}

\section{Seizure Onset}

Electrophysiological results for all patients are summarized in Table 2 . For 7 of the 10 patients, the ictal activity of at least one clinically significant seizure type was detected at onset from an electrode on the TPC. For 2 patients in Group A (Cases 3 and 5), onset was widespread but included the TP. An example of a seizure starting in the TP and the corresponding electrode localization is depicted in Fig. 2. The subject had extensive subdural electrode coverage of the subfrontal and subtempotal cortex, including the ATL (Fig. 2A). The seizure started at a single electrode on the TPC (see TP06, Fig. 2B), and seizure activity at this electrode did not propagate for 8 seconds. The seizure then spread rapidly throughout all of the TPC and the superior temporal sulcus. There was late propagation to the amygdala, hippocampus, and orbitofrontal cortex (Fig. 2B).

Importantly, most Group B patients (seizure onset outside the TP) had evidence of hyperexcitability in the TPC, as evidenced by the presence of clinically silent seizure activity or early seizure spread to the TP. The patient in Case 8, for example, had clinically silent seizure activity arising from the TP electrodes. Each of these recordings lasted less than 6 seconds and was not associated with symptoms. This patient also had early spread of ictal activity to the TP within 5 seconds, which occurred before propagation to the mesial temporal structures (amygdala or hippocampus).

\section{Interictal Discharges}

All temporopolar electrode sites were evaluated for the presence of interictal epileptiform discharges (IEDs; Table 2). In total, 7 of the 10 patients had IEDs from the TPC. For Group A, 5 (71\%) of 7 patients had IEDs arising from the TP. In Group B, 2 (67\%) of 3 patients had IEDs arising from the TP. Thus, the presence of IEDs localized to the TPC was not necessarily associated with seizure onset from the TP.

\section{Neuroimaging Correlation}

Both interictal FDG-PET and MRI data were reviewed for all patients (Table 3). Hypometabolism was observed in all patients who underwent interictal FDG-PET (9 of 9; 1 patient did not have a PET study). Two neuroradiologists (T.M. and A.A.C.) blinded to all clinical data (including the presence of IEDs, ictal onset, and FDG-PET results) reviewed preoperative MRI studies for all patients for evidence of MTS, temporopolar abnormal MRI signal, and anatomical lesions. Ultimately, 5 of 10 patients had evidence of MTS on neuroimaging. In Group A, 3 (43\%) of 7 patients had MRI evidence of MTS; in Group B, 2 (67\%) 
A

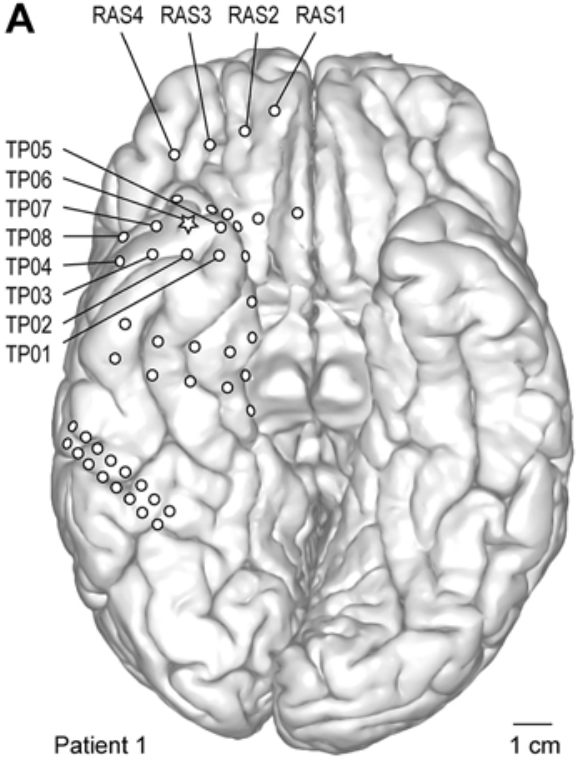

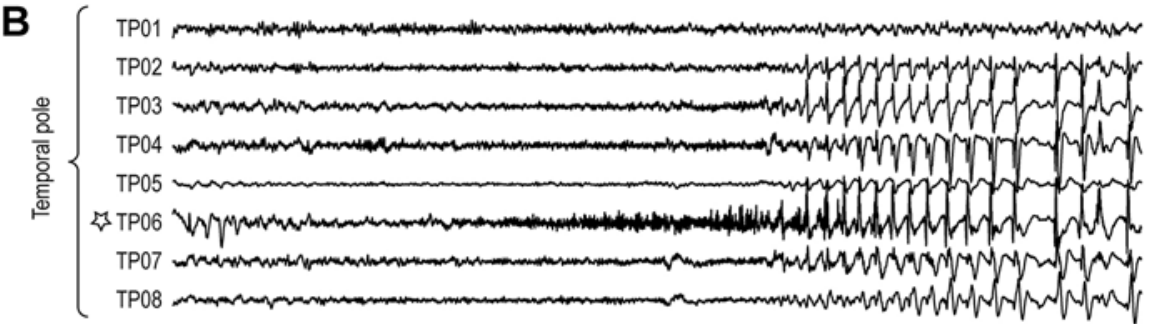
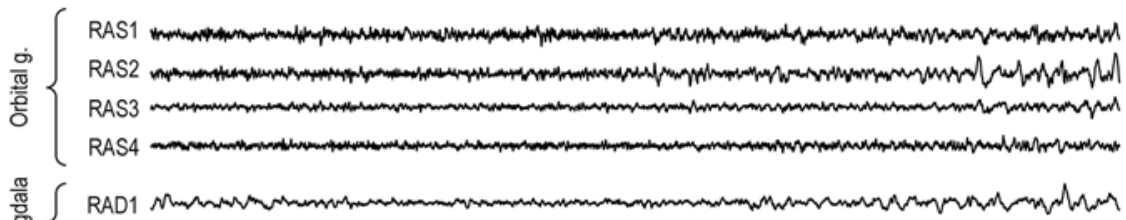

蜜 $\{$ RAD2 $200 \mu \mathrm{V}$ L

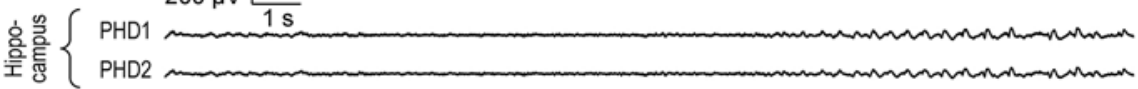

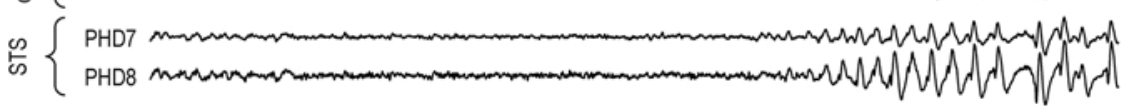

FIG. 2. Case 1. A: Anatomical reconstruction of the ventral brain surface demonstrating the location of implanted epicortical electrode arrays in a representative patient. Star denotes the recording site where seizure onset was observed (TP06). B: Example recordings from epicortical electrodes implanted over the TP and orbital gyrus (g.) and depth electrodes implanted in the amygdala, hippocampus, and superior temporal sulcus (STS). $s=$ second.

of 3 patients had MRI evidence of MTS. Three patients (Cases 5, 6, and 9) had abnormalities of the TPC apparent to the neuroradiologist on MRI. Of the patients with discernable TP abnormalities, 2 were in Group A (2 [29\%] of 7) and 1 was in Group B (1 [33\%] of 3). Detection of TP abnormalities varied slightly between the neuroradiologists, with an additional patient with TP abnormality being detected by a single neuroradiologist. Two examples of TP abnormalities detected on MRI are depicted in Fig. 3. In each example, there is high FLAIR signal of TPC and underlying white matter.

Neuroradiologists also examined MRI for structural lesions. One of the neuroradiologists determined that 3 patients had structural lesions evident on MRI: 2 (29\%) of 7 in Group A and 1 (33\%) of 3 in Group B. The other neuroradiologist determined that 6 of 10 patients had structural lesions evident on MRI: 5 (71\%) of 7 in Group A and 1 (33\%) of 3 in Group B. Magnetic resonance imaging in the patient in Case 4 demonstrated an intrinsic nonenhancing heterogeneously high T2 signal mass in the right mesial temporal lobe, consistent with a dysembryoplastic neuroepithelial tumor (DNT). Magnetic resonance imaging in Case 7 demonstrated a mesial temporal mass involving the hippocampus and amygdala, suggestive of

TABLE 3. Summary of FDG-PET, MRI, and pathological analysis data

\begin{tabular}{|c|c|c|c|c|c|c|c|c|c|c|c|}
\hline \multirow{2}{*}{$\begin{array}{l}\text { Case } \\
\text { No./ } \\
\text { Group }\end{array}$} & \multirow{2}{*}{$\begin{array}{c}\text { PET } \\
\text { Hypometabolism }\end{array}$} & \multirow{2}{*}{$\begin{array}{l}\text { PET } \\
\text { Laterality }\end{array}$} & \multicolumn{2}{|c|}{$\begin{array}{l}\text { MRI } \\
\text { MTS }\end{array}$} & \multicolumn{2}{|c|}{$\begin{array}{c}\text { MRI TP } \\
\text { Abnormalities } \\
\end{array}$} & \multicolumn{2}{|c|}{$\begin{array}{c}\text { MRI } \\
\text { Anatomical } \\
\text { Lesions } \\
\end{array}$} & \multirow[b]{2}{*}{ Lesion Location } & \multirow[b]{2}{*}{ Pathology } & \multirow{2}{*}{$\begin{array}{l}\text { Pathology } \\
\text { MTS }\end{array}$} \\
\hline & & & NR1 & NR2 & NR1 & NR2 & NR1 & NR2 & & & \\
\hline $1-A$ & Yes & Rt & - & - & - & - & - & + & Rt amygdala enlargement & FCD Ila & No \\
\hline $2-A$ & Yes & Bilat & - & - & - & - & - & + & Lt amygdala enlargement & FCD lb & No \\
\hline $3-A$ & Yes & $\mathrm{Lt}$ & $\mathrm{Lt}$ & Lt & - & - & - & - & NA & FCD Ila & No \\
\hline $4-\mathrm{A}$ & NA & NA & - & - & - & - & + & + & $\begin{array}{l}\text { Rt amygdala, periamygdaloid \& tempo- } \\
\text { ral piriform cortex, hippocampal head }\end{array}$ & DNT & No \\
\hline $5-A$ & Yes & Rt & Rt & Rt & Rt & Rt & - & - & NA & FND & Yes \\
\hline $6-A$ & Yes & $\mathrm{Lt}$ & - & - & $\mathrm{Lt}$ & $\mathrm{Lt}$ & - & + & Lt amygdala \& hippocampal head & FCD lb & No \\
\hline $7-A$ & Yes & $\mathrm{Lt}$ & $\mathrm{Lt}$ & Lt & - & - & + & + & Lt PHG & FCD IIIa & Yes \\
\hline $8-B$ & Yes & Rt & - & - & - & - & + & + & Rt fusiform gyrus & GG, FCD IIIb & No \\
\hline $9-B$ & Yes & $\mathrm{Lt}$ & $\mathrm{Lt}$ & $\mathrm{Lt}$ & - & $\mathrm{Lt}$ & - & - & Lt hippocampal sclerosis & None & Yes \\
\hline $10-B$ & Yes & $\mathrm{Lt}$ & $\mathrm{Lt}$ & $\mathrm{Lt}$ & - & - & - & - & Lt hippocampal sclerosis & None & Yes \\
\hline
\end{tabular}

+ = present; - = not present; FCD = focal cortical dysplasia; FND = focal nodular dysplasia; GG = ganglioglioma; NR1, NR2 = indicate interpretation by each of 2 neuroradiologists; $\mathrm{PET}=$ positron emission tomography; $\mathrm{PHG}=$ parahippocampal gyrus. 

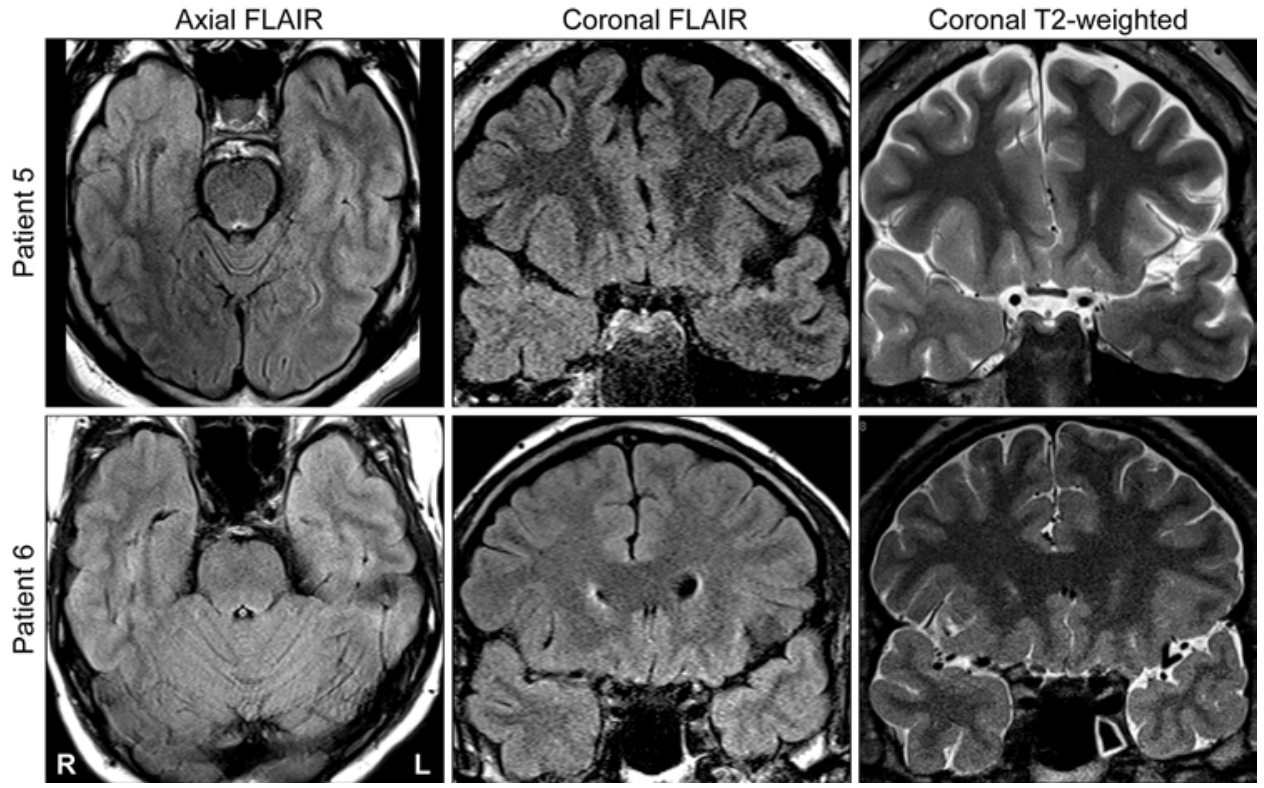

FIG. 3. Neuroimaging findings in 2 representative patients (Case 5, upper; and Case 6, lower). Axial FLAIR, coronal FLAIR, and coronal T2-weighted MR images obtained in each patient, showing clear atrophy and FLAIR signal abnormality of the TPC.

cortical dysplasia. Magnetic resonance imaging in Case 8 revealed an intrinsic T1 isointense and T2 hypointense lesion thought to represent a cavernoma (later diagnosed as a ganglioglioma). Other detected anatomical lesions involved subtle FLAIR signal change in the amygdala, which may explain why these abnormalities were not detected by both neuroradiologists.

\section{Pathological Correlation}

Pathological diagnosis was performed by a neuropathologist (P.K.), and the ILAE classification system was applied when appropriate (Table 3). All patients in Group A had focal nodular dysplasia (Case 5) or focal cortical dysplasias that could be classified by the ILAE classification system. In contrast, only 1 patient in Group B had a focal cortical dysplasia (Case 8, FCD Type IIIb and concomitant ganglioglioma). Two tumors were diagnosed: a ganglioglioma in Case 8 (Group B) and a DNT in Case 4 (Group A). In Group A, 2 of 7 patients had pathologically confirmed MTS; in Group B, 2 of 3 patients had pathologically confirmed MTS.

\section{Clinical Outcomes}

Clinical outcomes are summarized in Table 1. The average follow-up duration for all patients was 31.4 months. The average follow-up for Group A was 26.7 months and for Group B 42.3 months. Patient outcomes were classified using the Engel classification. In Group A, 4 (57\%) of 7 patients had a Class I outcome, $2(29 \%)$ of 7 had a Class II outcome, and $1(14 \%)$ of 7 had a Class III outcome. In Group B, 2 (67\%) of 3 patients had a Class I outcome and $1(33 \%)$ of 3 had a Class II outcome.

\section{Discussion}

The goal of this study was to delineate the role of the TP in TLE seizure networks. Understanding its role is crucial as the indications for CAH and SAH vary widely among epilepsy centers and exclusion of the TP from resection could be a cause of poor seizure freedom rates in patients who undergo SAH. ${ }^{9}$ The superiority of CAH over SAH for medically intractable TLE is a topic of intense debate; however, for patients with findings suggestive of MTLE, several studies indicate that $\mathrm{CAH}$ and $\mathrm{SAH}$ are equivalent with regard to seizure freedom. ${ }^{18,24,29,31,33}$ However, other studies have demonstrated better seizure outcomes with CAH compared with SAH, particularly in pediatric patients. ${ }^{4,10,31}$ Previous studies have revealed that even in patients with noninvasive imaging findings suggestive of MTLE, the TP can still be part of the epileptogenic zone. ${ }^{9}$ Thus, understanding when the TP is part of the epileptogenic zone should be an integral part of treatment planning to achieve an appropriate surgical intervention for patients with medically intractable TLE.

Despite any controversy regarding the indications for TP resection in patients with medically intractable TLE, numerous studies have suggested evaluating the role of the TP as part of the epileptogenic zone in patients with TLE. $6,9,20,21,28$ Though the amygdala, hippocampus, and parahippocampal gyrus are frequently implicated as the major players in many patients with TLE, histopathological alterations outside these structures occur frequently in those with TLE. 17,26,27 Additionally, MRI studies in patients with TLE have demonstrated abnormal anterior temporal T2 signal, ${ }^{26}$ gray-white matter signal blurring in the TP, ${ }^{17}$ and volume loss in the TP. ${ }^{13}$ The histopathological correlates of these imaging abnormalities remain unclear, though a variety of pathological abnormalities have been observed in the TP in patients with TLE. ${ }^{13,26,27}$ In 2012, Garbelli et al. ${ }^{17}$ compared 7-T MRI and myelin staining of TLE specimens and found reduced axonal density in the TP of patients with temporopolar blurring on MRI. Additionally, TP hypometabolism as evidenced by FDG-PET is frequently seen in patients with TLE. ${ }^{30}$ Taken together, 
these findings suggest that the TP can be significantly affected in TLE. As others have pointed out, ${ }^{7}$ it is important to note that though the TP may be affected in TLE, structural changes in the TP do not confirm its involvement in the epileptogenic zone.

That said, there is also electrophysiological evidence that the TP can play a crucial role in epileptogenesis in patients with TLE. For example, stimulation of the TP can be just as effective as stimulation of the amygdala, hippocampus, or parahippocampal gyrus in inducing seizures in TLE patients. ${ }^{22}$ Additionally, simultaneous onset of seizures in the amygdala, hippocampus, and hippocampal gyrus is a common phenomenon in TLE, ${ }^{9,21}$ as we address in detail below.

The main finding of this study is that the TPC plays a key role in the ictal onset of clinically significant seizures in a large proportion (70\%) of our patients with TLE. The patients who exhibited ictal onset from the TP were not those expected to have TP involvement at seizure onset based on noninvasive preoperative workup. Two blinded neuroradiologists searched for both structural abnormalities of the TPC on MRI and anatomical lesions in the temporal lobe (Table 3). Review of MRI by these neuroradiologists did not uniformly implicate the TPC in the epileptogenic zone. For example, only 2 of 7 patients in Group A were found to have an abnormality of the TP on MRI by both of our neuroradiologists. It is interesting to note that one of our neuroradiologists found that 5 of 7 patients in Group A had anatomical lesions, while our other neuroradiologist found anatomical lesions in only 2 of 7 patients, thereby illustrating the potential for discrepancy in MRI interpretation even by trained neuroradiologists. In contrast, 1 Group B patient (Case 9) was found to have a structural abnormality in the TP on MRI and did not demonstrate any spread of seizure activity to the TPC (with onset in the amygdala). These examples illustrate the difficulty in determining whether the TP should be considered part of the epileptogenic zone by noninvasive means. This fact is also apparent from our FDG-PET findings: every patient in our study who underwent FDG-PET (9 patients; Table 2) demonstrated hypometabolism of the TP, yet only 6 had ictal onset from the TP. Neither did the FDG-PET hypometabolism seem to correlate with the presence of interictal discharges from the TP. Ultimately, the absence of correlation between MRI and FDG-PET would suggest the importance of intracranial recordings from the TP in cases in which the TP might be involved, which others have also advocated. $., 21,28$

Our finding of ictal onset from the TP in 70\% of TLE patients agrees with other intracranial recording studies of the TP in patients with TLE.,.$^{921}$ In 2005, Chabardès and colleagues ${ }^{9}$ described 48 patients with TLE who had undergone concurrent SEEG recordings from the TP and hippocampus prior to CAH. Twenty-three (48\%) of the 48 patients demonstrated involvement of the TP either before or concurrent with the hippocampus. Among those patients with TP involvement at seizure onset, 9 had MTS and 11 had TP abnormalities on MRI. This is an important distinction from our smaller series in which only 3 of 7 patients with TP involvement at seizure onset had MTS. Interestingly, Chabardès et al. ${ }^{9}$ also found that patients with TP involvement at seizure onset actually had bet- ter postoperative seizure freedom rates than the patients without TP involvement at seizure onset $(95 \%$ vs $72 \%$ Engel Class I). Importantly, 5 of 7 patients in Group A had seizure onset in the TP concurrent with another temporal lobe structure. While it is possible that recording techniques with improved temporal resolution (for example, depth recordings of TP) could show onset at a specific site (rather than 2 concurrent sites), the finding of concurrent onset in the TP demonstrates that the TP plays an intimate role at seizure onset even with distant sites within the temporal lobe.

It is interesting to note that all Group A patients were retrospectively found to have pathological abnormalities in the anterior temporal cortex (Table 3), particularly cortical dysplasia. This finding is understandable given that ictal onset involved the TPC in each of these patients. Additionally, our results support the notion that the TP is more frequently involved at the pathological and electrophysiological levels in patients with dysplastic lesions of the mesial temporal lobe as opposed to other etiologies. Importantly, as discussed above, the TPC would not necessarily have been implicated in the epileptogenic zone based on MRI alone. Furthermore, imaging abnormalities can be seen in TPC in TLE patients regardless of whether onset involves the TP. ${ }^{17,26,27}$

This study has several limitations that must be considered. A major limitation is the small sample size that precludes the meaningful use of statistics. Additionally, there is potential for selection bias as patients with TLE and concordant video EEG, FDG-PET, and MRI findings (that is, MTS) undergo CAH without intracranial recording studies (and would not be included in this study). Given previous studies, ${ }^{9}$ we suspect that some patients with MTS exhibit seizures that involve the TP at seizure onset; however, we did not evaluate this possibility. Furthermore, our study population is quite heterogeneous, with several of our patients retrospectively found to have cortical dysplasias or other cortical lesions. With those limitations in mind, the present results still demonstrate the frequent possibility that the TP is involved in the epileptogenic zone in TLE patients where it would not usually be suspected.

Additionally, since all of our patients underwent $\mathrm{CAH}$, our study did not evaluate the relative benefits of CAH compared with SAH or selective laser ablation. It is possible that the similar outcomes seen with $\mathrm{CAH}$ and SAH occur because the TP is partially disconnected with certain approaches to the mesial temporal lobe. Given our findings, it is possible that the lower seizure freedom rates observed with LITT $^{23}$ result from the preserved connections to TPC. Future studies are needed to determine whether tailoring resection strategies to electrophysiological findings in the TP has value. Furthermore, it is possible that our finding of early involvement of the TPC is a nonspecific finding and also occurs in extratemporal epilepsies. The TP has extensive connectivity with brain structures outside the temporal lobe that would also support this possibility. ${ }^{20}$ However, given previous studies, ${ }^{9}$ this does not seem likely.

\section{Conclusions}

Using dense intracranial recordings of the TP, we demonstrated the ictal onset of clinically significant seizures 
from the TP in 7 of 10 consecutive patients with medically intractable TLE. Our findings provide evidence that the TP should be given consideration as part of the epileptogenic zone in patients with TLE even when noninvasive imaging studies (for example, MRI) would not suggest a role for the TP, particularly when dysplastic lesions are suspected. The presence of MRI abnormalities, FDG-PET hypometabolism, and IEDs are not necessarily associated with the presence of ictal onset from the TP. Involvement of the TPC in the epileptogenic zone may be a potential reason for poor seizure outcomes after selective mesial resections, particularly after LITT.

\section{Acknowledgments}

We acknowledge Eishi Asano, MD, PhD, for his insightful comments during the preparation of this manuscript. We thank Tammy Bryant, REEGT, for her assistance with data analysis.

This work was supported by the National Institutes of Health (Grant No. F32-NS087664).

\section{References}

1. Abel TJ, Rhone AE, Nourski KV, Ando TK, Oya H, Kovach $\mathrm{CK}$, et al: Beta modulation reflects name retrieval in the human anterior temporal lobe: an intracranial recording study. J Neurophysiol 115:3052-3061, 2016

2. Abel TJ, Rhone AE, Nourski KV, Granner MA, Oya H, Griffiths TD, et al: Mapping the temporal pole with a specialized electrode array: technique and preliminary results. Physiol Meas 35:323-337, 2014

3. Acar G, Acar F, Miller J, Spencer DC, Burchiel KJ: Seizure outcome following transcortical selective amygdalohippocampectomy in mesial temporal lobe epilepsy. Stereotact Funct Neurosurg 86:314-319, 2008

4. Bate H, Eldridge P, Varma T, Wieshmann UC: The seizure outcome after amygdalohippocampectomy and temporal lobectomy. Eur J Neurol 14:90-94, 2007

5. Blümcke I, Thom M, Aronica E, Armstrong DD, Vinters HV, Palmini A, et al: The clinicopathologic spectrum of focal cortical dysplasias: a consensus classification proposed by an ad hoc Task Force of the ILAE Diagnostic Methods Commission. Epilepsia 52:158-174, 2011

6. Bonilha L, Martz GU, Glazier SS, Edwards JC: Subtypes of medial temporal lobe epilepsy: influence on temporal lobectomy outcomes? Epilepsia 53:1-6, 2012

7. Caboclo LO, Garzon E, Oliveira PA, Carrete H Jr, Centeno RS, Bianchin MM, et al: Correlation between temporal pole MRI abnormalities and surface ictal EEG patterns in patients with unilateral mesial temporal lobe epilepsy. Seizure 16:816,2007

8. Chabardès S, Kahane P, Minotti L, Hoffmann D, Benabid AL: Anatomy of the temporal pole region. Epileptic Disord 4 (Suppl 1):S9-S15, 2002

9. Chabardès S, Kahane P, Minotti L, Tassi L, Grand S, Hoffmann D, et al: The temporopolar cortex plays a pivotal role in temporal lobe seizures. Brain 128:1818-1831, 2005

10. Clusmann H, Kral T, Gleissner U, Sassen R, Urbach H, Blümcke I, et al: Analysis of different types of resection for pediatric patients with temporal lobe epilepsy. Neurosurgery 54:847-860, 2004

11. Clusmann H, Schramm J, Kral T, Helmstaedter C, Ostertun B, Fimmers R, et al: Prognostic factors and outcome after different types of resection for temporal lobe epilepsy. J Neurosurg 97:1131-1141, 2002

12. Cohen-Gadol AA, Spencer DD: Use of an anteromedial subdural strip electrode in the evaluation of medial temporal lobe epilepsy. Technical note. J Neurosurg 99:921-923, 2003
13. Coste S, Ryvlin P, Hermier M, Ostrowsky K, Adeleine $\mathrm{P}$, Froment JC, et al: Temporopolar changes in temporal lobe epilepsy: a quantitative MRI-based study. Neurology 59:855-861, 2002

14. Ding SL, Van Hoesen GW, Cassell MD, Poremba A: Parcellation of human temporal polar cortex: a combined analysis of multiple cytoarchitectonic, chemoarchitectonic, and pathological markers. J Comp Neurol 514:595-623, 2009

15. Drane DL, Loring DW, Voets NL, Price M, Ojemann JG, Willie JT, et al: Better object recognition and naming outcome with MRI-guided stereotactic laser amygdalohippocampotomy for temporal lobe epilepsy. Epilepsia 56:101113, 2015

16. Elsharkawy AE, Alabbasi AH, Pannek H, Oppel F, Schulz R, Hoppe M, et al: Long-term outcome after temporal lobe epilepsy surgery in 434 consecutive adult patients. J Neurosurg 110:1135-1146, 2009

17. Garbelli R, Milesi G, Medici V, Villani F, Didato G, Deleo $\mathrm{F}$, et al: Blurring in patients with temporal lobe epilepsy: clinical, high-field imaging and ultrastructural study. Brain 135:2337-2349, 2012

18. Hu WH, Zhang C, Zhang K, Meng FG, Chen N, Zhang JG: Selective amygdalohippocampectomy versus anterior temporal lobectomy in the management of mesial temporal lobe epilepsy: a meta-analysis of comparative studies. J Neurosurg 119:1089-1097, 2013

19. Josephson CB, Dykeman J, Fiest KM, Liu X, Sadler RM, Jette N, et al: Systematic review and meta-analysis of standard vs selective temporal lobe epilepsy surgery. Neurology 80:1669-1676, 2013

20. Kahane P, Bartolomei F: Temporal lobe epilepsy and hippocampal sclerosis: lessons from depth EEG recordings. Epilepsia 51 (Suppl 1):59-62, 2010

21. Kahane P, Chabardès S, Minotti L, Hoffmann D, Benabid $\mathrm{AL}$, Munari C: The role of the temporal pole in the genesis of temporal lobe seizures. Epileptic Disord 4 (Suppl 1):S51S58, 2002

22. Kahane P, Tassi L, Francione S, Hoffmann D, Russo GL, Munari C: Manifestations électrocliniques induites par la stimulation électrique intracérébrale par «chocs dans les épilepsies temporales. Clin Neurophysiol 23:305-326, 1993

23. Kang JY, Wu C, Tracy J, Lorenzo M, Evans J, Nei M, et al: Laser interstitial thermal therapy for medically intractable mesial temporal lobe epilepsy. Epilepsia 57:325-334, 2016

24. Kuang Y, Yang T, Gu J, Kong B, Cheng L: Comparison of therapeutic effects between selective amygdalohippocampectomy and anterior temporal lobectomy for the treatment of temporal lobe epilepsy: a meta-analysis. Br J Neurosurg 28:374-377, 2014

25. Lüders HO: Textbook of Epilepsy Surgery. Boca Raton: CRC Press, 2008

26. Meiners LC, Witkamp TD, de Kort GA, van Huffelen AC, van der Graaf Y, Jansen GH, et al: Relevance of temporal lobe white matter changes in hippocampal sclerosis. Magnetic resonance imaging and histology. Invest Radiol 34:38-45, 1999

27. Mitchell LA, Jackson GD, Kalnins RM, Saling MM, Fitt GJ, Ashpole RD, et al: Anterior temporal abnormality in temporal lobe epilepsy: a quantitative MRI and histopathologic study. Neurology 52:327-336, 1999

28. Munari C, Hoffmann D, Francione S, Kahane P, Tassi L, Lo Russo G, et al: Stereo-electroencephalography methodology: advantages and limits. Acta Neurol Scand Suppl 152:5669, 1994

29. Nascimento FA, Gatto LA, Silvado C, Mäder-Joaquim MJ, Moro MS, Araujo JC: Anterior temporal lobectomy versus selective amygdalohippocampectomy in patients with mesial temporal lobe epilepsy. Arq Neuropsiquiatr 74:35-43, 2016

30. Rubin E, Dhawan V, Moeller JR, Takikawa S, Labar DR, 
Schaul N, et al: Cerebral metabolic topography in unilateral temporal lobe epilepsy. Neurology 45:2212-2223, 1995

31. Schramm J: Temporal lobe epilepsy surgery and the quest for optimal extent of resection: a review. Epilepsia 49:12961307,2008

32. Tanriverdi T, Olivier A, Poulin N, Andermann F, Dubeau F: Long-term seizure outcome after mesial temporal lobe epilepsy surgery: corticalamygdalohippocampectomy versus selective amygdalohippocampectomy. J Neurosurg 108:517524, 2008

33. Wendling AS, Hirsch E, Wisniewski I, Davanture C, Ofer I, Zentner J, et al: Selective amygdalohippocampectomy versus standard temporal lobectomy in patients with mesial temporal lobe epilepsy and unilateral hippocampal sclerosis. Epilepsy Res 104:94-104, 2013

34. Wiebe S, Blume WT, Girvin JP, Eliasziw M: A randomized, controlled trial of surgery for temporal-lobe epilepsy. N Engl J Med 345:311-318, 2001

35. Willie JT, Laxpati NG, Drane DL, Gowda A, Appin C, Hao $\mathrm{C}$, et al: Real-time magnetic resonance-guided stereotactic laser amygdalohippocampotomy for mesial temporal lobe epilepsy. Neurosurgery 74:569-585, 2014

\section{Disclosures}

The authors report no conflict of interest concerning the materi- als or methods used in this study of the findings specified in this paper.

\section{Author Contributions}

Conception and design: Abel, Kawasaki, Howard, Werz. Acquisition of data: Abel, Woodroffe, Moritani, Capizzano, Werz, Kirby. Analysis and interpretation of data: Abel, Kawasaki, Werz, Kirby. Drafting the article: Abel, Woodroffe, Nourski, Werz. Critically revising the article: Abel, Woodroffe, Nourski, Werz, Kirby. Reviewed submitted version of manuscript: Abel, Woodroffe, Capizzano, Kawasaki, Howard, Werz. Approved the final version of the manuscript on behalf of all authors: Abel. Administrative/ technical/material support: Howard. Study supervision: Howard, Werz.

\section{Supplemental Information \\ Previous Presentations}

Portions of this work were presented at the 2016 Congress of Neurological Surgeons Annual Meeting held in San Diego, California, on September 24-28, 2016, and the American Epilepsy Society Annual Meeting held in Houston, Texas, on December 2-6, 2016.

\section{Correspondence}

Taylor J. Abel, Department of Neurosurgery, University of Iowa Hospitals and Clinics, 200 Hawkins Dr., Iowa City, IA 52242. email: taylor-abel@uiowa.edu. 